\section{A a} ON

\section{GASTRIC HAEMORRHAGE.}

\author{
Given before the Exeter Division \\ BY
}

CHARLES MILLER, C.B.E., M.D., F.R.C.P.,

CONJUlTING PHYSICIAN TO THE LONDON hoSPITAL; ASSISTANT PHYSICIAN, ROYAL DEVON AND EXETER HOSPITAL.

Few occurrences cause so much consternation as the vomiting of a large quantity of blood. The equally serious but less sensational events, fainting, sudden pallor, and the subsequent passage of a tarry stool, do not produce the same effect on the minds of the patient and his friends. But from the standpoint of the doctor the two events are seen to be of equal importance, for they point to internal haemorrhage in stomach or duodenum. For convenience I propose to consider the acid part of the duodenum as part of the stomach, as both are subject to the same kind of digestive juice. Pathologically the two regions are subject to similar disorders, though anatomically they have to be described separately.

It used to be thought that melaena without haematemesis was a point in favour of the diagnosis of duodenal ulcer rather than of gastric ulcer. But this condition is found in cases of gastric ulcer, and, on the other hand, haematemesis can occur with duodenal ulcer. In dealing briefly with gastric haemorrhage only copious haomorrhage will be considered. Occult blood in the stools has its value in the diagnosis of certain alimentary disorders, but is outside the subject of this occasion.

At the beginning of the century haematemesis, except in cases of cirrhosis of the liver, was thought to be a sure sign of gastric ulcer. By gastric ulcer was meant a large ulcerated area, visible and palpable in the exposed stomach. The advance in gastric surgery has proved many ideas about clisorders of the stomach to be wrong, and gastric haemorrhage has been shown to be due to many conditions other than a gross ulcer of the stomach. It may be said that, although a gastric ulcer can be associated with copious haemorrhage, many large chronic ulcers persist for years without any noticeable bleeding, and many cases of copious haemorrhage are found among patients who have no sign of ulcer of any size.

\section{Causes of Gastric Haemorrhage.}

As a general principle it may be stated that when haemorrhage occurs there must have been loss of tissue -that is, ulceration. The ulceration may be minute and even microscopic. Gastric ulceration is due to the digestion of the stomach wall by the gastric juice. But beforo self-digestion takes place there is some damage to the stomach wall, for gastric juice does not digest healthy mucous membrane.

Gastric ulceration is due to the digestion of damaged tissue in the wall of the stomach. The wall of the stomach is well supplied with blood, and during digestion it has the appearance of soft red velvet, which looks as if it would bleed freely if scratched. The blood should, and does, remain in the blood vessels; but if there is any damaged tissue the gastric juice will digest it, and may open a blood vessel or vessels. Of course, trauma may directly produce haemorrhage. The act of violent vomiting, the passage of a tube into the stomach, a blow on the epigastrium, may produce haemorrhage from a stomach that is not ulcerated even minutely, and the damage done by severo trauma can produce subsequent ulceration. But haemorrhage from the stomach is usually a sequel to the digestion of the mucous membrane and deeper parts of the stomach wall by the action of the gastric juice.

It becomes necessary to examine the various processes by which the stomach can be damaged. Trauma has already been mentioned. It is conceivable-in fact it has happened -that trauma producing submucous haemorrhage has been followed by chronic ulceration.

Corrosive poisons, of course, will damage the stomach and produco haemorrhage by their own action; I ean remember a case of repeated haemorrhage coming from a chronio gastric ulcer following attempted suicide by spirits of salt some years before. But such cases as these are few and not worth further consideration at the moment.

Apart from such traumatic causes, other possible causes of damage to the stomach have to be considered. First; interruption of the blood supply will undoubtedly lead to the death of tissue locally, and the gastric juice will do the rest.

In cases of mitral stenosis when, as a terminal event, auricular emboli are showered into the arterial stream some may block up vessels in the wall of tho stomach. The ne:ghbouring mucous membrane is starved of blood and in its damaged state can be digested. The digestion may go further than the block in the arteriole and haemorrhage can follow. This is a terminal event, and sometimes the stomach is found post mortem to be filled with blood. An example of this cause of gastric haemorrhage was afforded by the case of a man, aged 26 , who died from mitral stenosis. Post mortem there wero recent infarcts in the spleen and kidneys; the stomach was full of blood; there were numerous small ulcers throughout all parts of the stomach. Microscopic examination showed blocking of some arterioles and a thrombus in a branch of the coronary artery. In one section the artery was empty and its wall deficient at one point so that the lumen communicated with the cavity of the stomach.

In cases of progressive endocarditis gastric haemorrhage is sometimes seen. In these cases also emboli are the cause, but here the emboli may contain microbes and can produce the effects of acute local inflammation. A woman, aged 51, died from ulcerative endocarditis of the acrtic and mitral valves. Post mortem in the stomach there were many small punched-out ulcers situated in greatest numbers in the fundus. There was some blood in the stomach. Arterial emboli can be spread throughout the stomach, but are usually most numerous in the fúndus, which is not often the site of gastric ulcer.

Obstruction of veins can also be followed by similar results. In the case of a boy aged 12 , who died of haematemesis, there was such proliferation of the intima of the reins that many sections showed complete blocking. Here there were numerous haemorrhagic points visible in the stomaich.

In general miliary tuberculosis, when the blood is soaking the tissues with tubercle bacilli, tubercles may spring up in the gastric wall and are promptly digested. Occasionally, but rarely, slight haemorrhage may occur from the bases of the digested tubercles.

Cirrhosis of the liver and other conditions which produce portal congestion can cause haemorrhage. The explanation is that the bleeding comes from the bursting of a full vein. But in such cases the circulation in the portal area is slowed, and stagnation results. Conceivably this state of affairs would help the gastric juice to effect some superficial ulceration, leading very easily to haemorrhage. Also it should be remembered that chronic gastric ulceration is not uncommon in cases of alcoholic cirrhosis of the liver.

Gastric haemorrhage is sometimes seen in patients who have a raised blood pressure. In such subjects haemorrhage can occur from any region in which the arteries do not derive much support from the tissues through which they are travelling. Nose-bleeding, cerebral haemorrhage, and gastric haemorrhage may occur. In the early days of gastric surgery when gastro-jejunostomy was the treatment of most gastric conditions, a man of 30 was admitted for haematemesis. Later gastro-jejunostomy was performed. He was readmitted for haematuria. He had a systolic blood pressure of $210 \mathrm{~mm}$. of mercury, retinal haemorrhages, and granular casts in the urine. Post mortem small fibrous kidneys were found.

Primary blood diseases frequently produce subcutaneous and other haemorrhage, and the stomach does not escape, but this form of haemerrhage need not be considered further.

The part that syphilis plays in the production of gastrio ulceration and haemorrhage can be best illustrated by the recital of two cases. A man aged 35, a schoolmaster, was admitted to hospital pale and collapsed after haematemesis. 
After he had recovered the abdomen was opened and several recent gummata were seen in the liver. The remainder of the liver appeared to be healthy. Certainly there was no great amount of fibrosis, and it could not be argued that portal congestion was an important factor. Possibly he had a syphilitic gastric ulcer which escaped notice. Another man was admitted in a similar state whose Wassermann reaction was found to be positive. He was given antisyphilitic treatment and did well. No abnormality was found radiologically. He went out and visited the seaside, where he died, on the pier, from haematemesis. Further there is McNee's classical case in which he found spirochaetes in the wall of a gastric ulcer.

The two cases I have quoted show that haematemesis can occur in the syphilitic, and McNee's case prores that syphilis can cause gastric ulceration. Possibly syphilitic ulceration is more frequent than is thought.

\section{Chronic Gastric Ulcer.}

So far, with the exception of cirrhosis, only the less common causes of gastric haemorrhage have been considered.

In chronic gastric ulcer a vessel of some size in the base or wall may be eroded. There is no difficulty here in appreciating how haemorrhage arises. The exposed ressel is eroded by the action of the gastric juice. Such an event occurs commonly in ulcers of the lesser curvature that become adherent to the pancreas. The splenic vein may be in the floor of the ulcer, and haemorrhage from it may be very copious. Of the chronic gastric ulcers this form is the one most commonly associated with severe bleeding.

\section{Caxcer of the Stomach.}

It is also easy to see how haemorrhage can occur with cancer of the stomach. The growth, as it spreads, contains much semi-necrotic tissue which is readily digested and ulcerated. Bleeding can occur, and usually does, but infrequently in large amount. Blood is often seen in the recovered test meal in cases of gastric carcinoma, and if it is present in all specimens of a fractional test meal the diagnosis of growth receives strong support. Also the repeated discovery of occult blood in the stools is an important diagnostic point in a suspected case. But a haematemesis that blanches the patient is not as common in carcinoma of the stomach as in cases of simple chronic ulceration.

\section{ACUTE UlCer.}

These disorders, however, do not provide more than half of the cases admitted to hospital for haematemesis or melaena. We are all familiar with the pale, plump young woman who vomits blood, and is admitted in a collapsed condition. But it will be the experience of most doctors that young women suffer less from gastric haemorrhage than they did fifteen years ago. Perhaps the greater freedom of limb and increased activity, and tho more hygienic dress of to-day, have improved the health of young women in this respect as in many others. Chlorosis is now a rare disease. Indigestion and chlorosis went hand in hand. The disappcarance of chlorosis is eontemporary with the smaller incidence of gastric haemorrhage in young women.

At one time, in the earliei days of gastric surgery, it was thought that these cases were surgical emergencies. The results were not satisfactory. As a rule nothing was found in the stomach except blood, and in many cases the operation was more than the patient could stand. Even at the post-mortem examination it was difficult to see where the haemorrhage had occurred. It is obvious that in such cases the bleeding must be from many sniall points. If we remember that there is a redundanes of mucous membrane in the stomach it is easy to imagino how a minute ulcer can he coverci over by the neighbouring mucous membrane.

When Matthes was attempting to produce artificial uleers in the stomachs of dogs, he found great difficulty in doing so. If he removed a small area of mucous niembrane the bare area was soon made invisible by the contraction of the smooth muscle and the covering made by the surrounding mucous membrane. He attempted to get orer this difficulty by suturing the muscular coat to a glass ring placed on the peritoneal surface. Even so the bare area was no further ulcerated, and the mucous membrane grew orer it. This shows that small ulcers are easily obliterated by the contraction of the underlying muscle and the new covering provided by the mucous membrane. Actually he was unable to produce a lasting ulcer until he had given large quantities of acid with the food.

\section{Gastrostaxis.}

The condition of gastric haemorrhage without any obvious ulceration or bleeding point has been called "gastrostaxis." There is a danger in coining rew words to describe morbid conditions, unless the word gives some explanation of the condition. There is no harm in using such a word as "gastrostaxis" if the user is merely describing a phenomenon, and does not think that he has given an explanation of the cause of the phenomenon.

I have attempted to argue that stomachs bleed if they digest their own wall so as to open blood vessels, and that to allow this process to begin some part of the wall of the stomach has previously been damaged and rendered necrotic. If we keep the term ulcer, either gastric $\mathrm{Or}^{\circ}$ duodenal, for something gross that can be seen and felt, we must have some other term for cases of microscopic or minute ulceration in which haemorrhage occurs. The haemorrhage may be the only clinical evidence of its presence, and post mortem, but for the haemorrhage, the stomach might pass as normal to the naked eye. The word "erosions" has been used to denote these minute and even microscopic ulcers. Even if they are just visible these small acute ulcers can be called erosions. It does not matter much to clinicians whether they are just visible or invisible, for it is to be hoped that they will never see during life the inside of a stomach in which there has been haemorrhage from "erosions."

These minute ulcers are best seen where lymphatic tissue is most thickly spread-that is, along the lesser curvature and in the distal part of the stomach. There are also smaller isolated lymphatic follicles scattered throughout the stomach. The lymphatic tissue of the stomach behaves in the same way as lymphatic tissue elsewhere. Infection of a nail-bed may cause enlargement of axillary glands and even an abscess. The lymphatic tissue of the stomach can behave in the same way, but there is the added factor of the digestive power of gastric juice; which will eat away any damaged tissue in the stomach and leave a hole. In the process the stomach may be perforated or blood vessels be eroded. Dr. Beaumont, in his historical description of the stomach of Alexis St. Martin, described what he saw through a gastric fistula. He sometimes saw raised red areas that were comparable to boils. Alexis was no total abstainer, nor was the popular press then in existence to direct his diet on hygienic lines. The result was that Dr. Beaumont wass able to look inside the stomach of a man who frequently ill-treated it.

If the lymphatic tissue of the stomach becomes inflamed as the result of infection it is incumbent on us to discover how the infection arose, and by what path the stomach was invaded. Obviously the mouth is a possible focus of infection. The condition of the teeth and gums in many dyspeptics is beyond description, and insistence on oral hygiene may remove gastric symptoms.

The acid gastric juice has an antiseptic action on many or'ganisms, but not on all, and it is possible for swallowed organisms to set up a gastric inflammation. Possibly also the lymphatics of the oesophagus may serve as a path for invading organisms. The following case illustrates the effect of oral sepsis.

An elderly man was treated by diathermy for cancer of the tongue. The tongue became very foul, and a large slough formed. About a week after the operation he had a large vomit of blood. There was no bleeding from the tongue, and there were no gastric symptoms in his past hisiory. The inference was that he had an acute septic infection of his stomach. He made a good recovery.

\section{Extragastric cadses of HaEmorrhage.}

But the source of infection may be in various situations. An inflamed gall bladder may be associated with haematemesis.

A woman, aged 22, was admitted with grave anaemia, following haematemesis. After going into her history and observing her, a diagnosis of cholecystitis was made. At the subsequent operation 
the gall bladder was found to contain pus and stones. Nothing was found wrong by external examination of the stomach. She was cured of her gastric symptons and had no further haemorrhage within two years.

Perhaps the most frequent extragastric cause of gastric haemorrhage is a fibrous deformed appendix, the result of past inflammation.

A boy of 10 had to get up one night with an urgent desire to defaecate. $\mathrm{He}$ was found outside the priy door, unconscious, with vomited blood issuing from his mouth. He was brought to hospital, and made a good recovery, without anyone being able to discover what was the matter wilh him. A year later he was readmitted blanched and pulseless, and he romited blood after

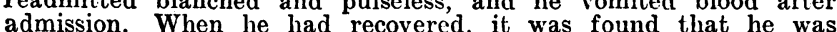
very tender on rectal examination high up to the right. On his previous stay in hospital it had been impossible to examine his rectum owing to his struggles. At the operation a thick constricted fibrous appendix was removed. The stomach and duodenum were pronounced to be healthy. There had been no repetition of his trouble two years after the operation.

Other cxamples of similar cases could be quoted to illustrate the effect of extragastric disorders on the stomach. There are well known dyspeptic conditions that are dependent on the presence of an unhealthy gall bladder or

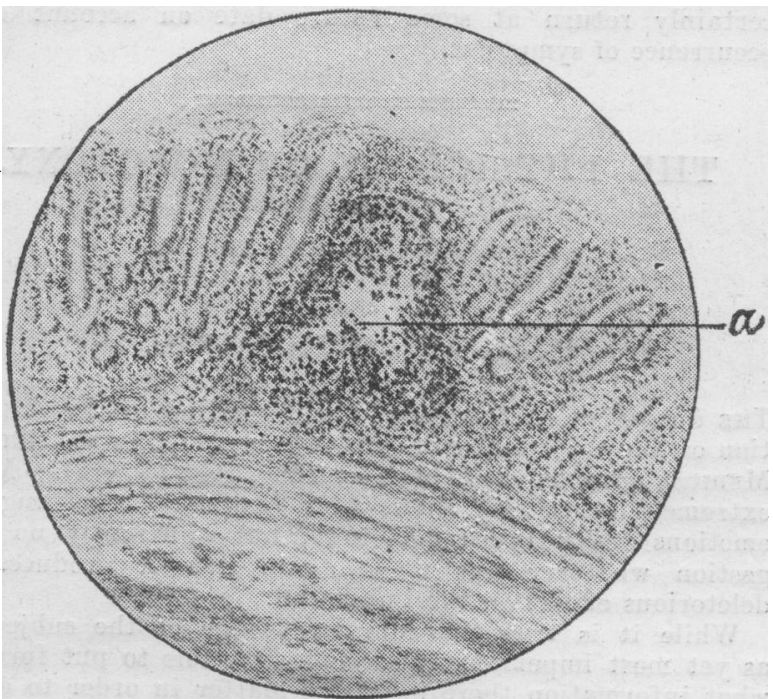

Two sections from the stomach of a girl who died from haematemesis without a visible ulcer being found. $a=1$ ymphatic follicle; $a^{\prime}=$ space
in the mucosa suggestive of a burst lymphatic follicle.

appendix, of which examples have been quoted. But the occurrence of haemorrhage would suggest an anatomical connexion by which an inflammatory process could extend from an inflamed viscus to the stomach. Such a pathway is most readily provided by the lymphatics. An upwardgoing lymphatic tract can be demonstrated starting from the ileo-caecal angle and extending in the region of the pylorus. The spread of tubercles can sometimes be traced along this path, and the appearances of acute inflammation have been noticed by surgeons during life.

There may be similar lymphatic paths by which the stomach is connected to other viscera in the peritoneal zavity. It is well known that gastric symptoms are associated with disorders of other abdominal organs. When a definite organic change, such as leads to haemorrhage from the stomach, occurs in association with an inflamed appendix or gall bladder, it can be argued that there is not only a disordered function of the stomach set up by an extragastric disorder, but-also a disordered structure which has been produced by an extension of the inflammatory process to the stomach. If such definite foci of infection as those of the appendix and gall bladder can be causes of gastric haemorrhage it is conceivable that such conditions as intestinal stasis with ptosis and other postural disorders can act in similar fashion. Very possibly such conditions as a "cesspool" colon can cause gastric ulceration and haemorrhage. Frequently gastric liaemorrhage occurs in the subjects of enteroptosis, and in some of them chronic gastric ulcer develops.

It can be stated as a fact that a large number of cases of gastric haemorrhage are found among those whose primary trouble is outside the stomach. It is possible, and even probable, that the originating cause of a visible gastrio ulcer lies outside the stomach, but this is a very difficult question which brings into consideration the degree of acidity of the gastric juice and other complicating factors. The following case is of interest and is suggestive.

A plump, constipated, dark young woman, aged 20 , was admitted to hospital very pale and collapsed after haematemesis. She rallied satisfactorily, and was put through every form of examination. Nothing positive was found, except that the acid of the test meal was very high. She went out in good health, and three months later was sent for and another test meal done. On this occasion the acidity was normal and she was free from gastric symptoms. Six month later she was readmitted, blanched and ymptoms. Six months later she was readmitted, blanched and pulseless, having vomited a lot of blood. She continued to vomit of the absence of any definite evidence, it was thought advisable to explore. A small duodenal ulcer was found. The ulcer was obviously recent and, as a matter of opinion, not older than a few weeks. Perhaps it would be safer to say that it was not a chronic ulcer.

It was thought that in this caso hacmorrhage had occurred as a result of an acute inflammation of the stomach, and that the small ulcers had healed; with the exception of the one found in the duodenum. One thing is

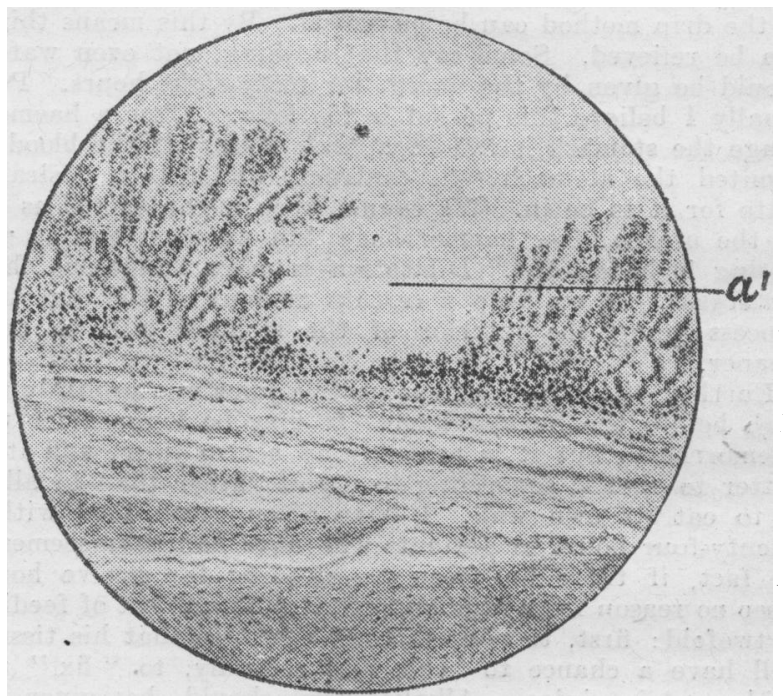

certain-namely, the small ulcer found at operation was not the cause of her first haemorrhage.

\section{Sumpary as to Causation.}

To sum up, gastric haemorrhage comes from gastrio ulceration. The ulceration may be gross, or microscopic, or just visible-that is, it may be chronic or acute, and more frequently than is usually thought, it is acute and microscopic. In the majority of cases the ulceration follows infection and inflammation. Further, the origin of the trouble often lies outside the stomach.

\section{Diagrosis and Treatment.}

When we are faced by a patient who has recently vomited a large quantity of blood we have to treat the patient rather than the disease. It is one of the conditions in which we must treat first and diagnose later, if we can. Before wo start treatment wo have to consider what our aims are. We wish to improve the patient's condition, stop the haemorrhage, and prevent its recurrence. At the outset of treatment no surgical measure can be consiclered. First we want to ensure rest and quiet for the patient, and relief for his anxiety. The patient must be moved as little as possible; if he has to be moved to hospital, the journey should be delayed until he is well under morphine.

Morphine should be given at once, and the patient should be kept under morphine, to which atropine can be added. Perhaps after the initial dcse of morphine, omnopon-scopolamine may seem advisable rather than repeated morphine. Collapse must not be too drastically treated. A certain amount of collapse is wanted so as to lower the chances of further haemorrhage. On no account 
hould transfusion be performed, nnless a surgeon is opening the abdomen.

We have to make some sort of preliminary diagnosis. If the patient is young and well covered the condition of the stomach is probably not a chronic ulcer, and the treatment must be medical. If a history can be obtained which suggests chronic gastric or duodenal ulcer, then a surgeon should be consulted, not with a view of immediate operation, but so that he will be able to appreciate any subsequent change in the patient. If, in such a case, there is a recurrence of haemorrhage and it is thought that the haemorrhage is coming from some such vessel as the splenic vein, then transfusion of blood and exploration can take place together. But the majority of cases of gastric haemorrhage recover without the aid of the surgeon. Only in cases of repeated haemorrhage and when a chronic gastric ulcer is suspected should surgical measures be employed.

The most important question is the feeding of the patient. On this point there are various opinions. Certainly no one will object to rectal salines being given. If they are well tolerated they can be given every six hours, or the drip method can be employed. By this means thirst can be relieved. Some say that nothing, not even water, should be given by the mouth for forty-eight hours. Personally I believe this period is too long. After a haemorrhage the stomach is left in a slack state. If the blood is vomited the stomach will contract, which is a desirable state for it to be in. We cannot keep it contracted as we do the uterus after haemorrhage, but we must aim at not letting it be dilated. Dilatation is more dangerous than peristalsis. If we keep a certain amount of the digestive process going the interior of the stomach will be kept cleaner.

Further, the acidity of the gastric juice was probably ton high before the haemorrhage. It may be lower after the haemorrhage, but it is probably an active juice, and it is better to give it something to digest rather than to allow it to eat its own wall. I have often given food within twenty-four hours to patients who have had haematemesis. In fact, if there has been no bleeding for twelve hours 1 see no reason for withbolding food. The object of feeding is twofold: first, to nourish the patient so that his tissues will have a chance to heal; and secondly, to " fix" the acid gastric juice. Alkalis also should be given to neutralize the acidity of the gastric juice. While food and alkalis lower the acidity of the juice for a time, they kave the faculty of stimulating a further secretion; so that treatment consists in alternately neutralizing and stimulating the secretion. This means that the patient is constantly taking something, either food or medicine.

Two names are associated with the special dietetic methods of treating gastric ulcer-Lenhartz and Sippy. As they are respectively German and American, some small modifications are desirable in their diets to make them more suitable for British tastes. But apart from these slight modifications the original diet should be closely followed. Too often one hears that the patient was treated on the lines of Lenhartz or Sippy, but without success. Failure cannot be laid at the doors of Lenhartz or Sippy, for the line of treatment will be found to have no relation to that laid down by either of them. The details of these two diets need not be given here, but the Lenhartz method is more suitable for early feeding after haemorrhage.

Drugs, in the treatment of gastric haemorrhage, have their uses in promating rest and in lessening the activity of the gastric juice. First and most important is morphine. For peace of mind, for rest and stillness of the stomach, to allay the sensation of thirst, there is nothing to take the place of morphine. Belladonna or atropine, particularly by the mouth, lessen the secretion of gastric juice. Alkaline mixtures or powders should be given freely and regularly during treatment. The carbonates of magnesia, soda, and calcium can be used alternately with the feeds. Bismuth may be given at intervals of six hours.

Other measures have their advocates. On the analogy of the hypotonic uterus, washing out the stomach has been recommended. It is rather a trying ordeal for a collapsed patient, but possibly would do good to a patient whose stomach was distended with blood, which he was unable to vomit. However, unless a patient is dying, blood is usually vomited. Lavage then is not a method to be advised, as the passage of the tube is certain to cause tagging and straining, which will encourage further bleeding. Horse serum in various forms is reconmended, but its efficacy is doubtful. Calcium has even less to recommend it. Opinions differ about opening the bowels. Some advise that no attempt be made for a week. Personally I recommend an enema or a glycerin suppository at the end of the second day, and repeating the treatment on alternate days.

After the patient has recovered from the haemorrhage full investigation has to be made into the gastric condition. In some cases there is diffeulty in going further with the patient, as he may say that the haemorrhage has done him good. In some instances gastric acidity is lowered after haemorrhage, and, as many symptoms are dependent on this acidity, the patient feels better; rest in bed relieves the symptoms of many dyspeptics. But an energetic attempt should always be made to diagnose the condition which hạs led to gastric haemorrhage, for if the patient declines further treatment at the time he will almost certainly return at some future date on account of a recurrence of symptoms.

\section{THE PHYSIOLOGY OF MONOTONY.} BY

\author{
R. J. S. McDOWALE, D.Sc., F.R.C.P.Ev., \\ PROFESSOR OF PHYSFOLOGY, MnTG'S COLLEGE, LONDON; \\ AXD \\ H. M. Wells, Pr.D., B.Sc.
}

THE discussion at the Nottingham meeting of the Association on the subject of monotony was reported in the Britrs Medical Jounnal on September 11th, 1926 (p. 472). Many extremely interesting views were expressed regarding the emotions which cause monotony, but apparently no suggestion whatever was made as to how it produces its deleterious effects on the individual.

While it is true that our knowledge of the subject is as yet most imperfect, it appears desirable to put forward what information there is on the matter in order to assist in the experimental investigation of what is certainly an increasingly important industrial problem. Within recent years various workers in this department have carried out researches in relation to the integration of the circulation, and considerable evidence has accumulated which indicates that certain factors are clearly concerned.

Normally, whenever any physical effort is made the circulation at once adapts itself to the demands of the various parts of the body. During exercise a definite series of mechanisms is brought into play which causes the tissues in use to be supplied with more blood. In the first instance the metabolites of muscular activity, such as carbon dioxide and lactic acid, dilate the capillaries in the tissues being used, This has recently been made clear by the work of Hemingway and McDowall. In addition, impulses passing up the afferent nerves call into operation the Lovén reflexes by. which the larger vessels to the part are also dilated. At the same time the blood supply to unused parts of the body is reduced by the increased activity of the vasomotor centre. This centre has been shown by Dale and Evans to be controlled largely by carbon dioxide, and the production of this is, we know, increased in muscular exercise. Further, as a result of compression of the valvular veins, increased respiration, and the action of carbon dioxide on the venous reservoir-both directly and indirectly through the vasomotor centre-the venous pressure is increased and the heart accelerated. The total result is that in exercise the blood pressure is markedly increased.

But the adaptation of the circulation may begin before the actual commencement of the exercise. It has long been known that the heart of a sprinter is accelerated before the race, and that the respiration may be increased. Recently evidence has been obtained by Aveling, McDowall, and Wells that the blood supply of the skin, which is not called upon until sweating commences, is appreciably 